Pacific Journal of Mathematic 


\title{
SEMIDIRECT PRODUCT OF SEMIGROUPS IN RELATION TO AMENABILITY, CANCELLATION PROPERTIES, AND STRONG FØLNER CONDITIONS
}

\author{
MARIA KLAWE
}

\begin{abstract}
The purpose of this paper is to settle two problems. The first is Sorenson's conjecture on whether every right cancellative left amenable semigroup is left cancellative. The second, posed by Argabright and Wilde, is whether every left amenable semigroup satisfies the strong Fólner condition (SFC). We first show that these two problems are equivalent, then prove that the answer to both questions is no, through analyzing the semidirect product of semigroups in relation to amenability and cancellation properties. We conclude by investigating further the properties of semigroups satisfying SFC, and finally include some analogous results for left measurable semigroups.
\end{abstract}

1. Introduction. For any semigroup $S$, let $m(S)$ be the Banach space of bounded realvalued functions on $S$ with the sup norm. For each $s \in S$ we define a linear operator $\iota_{s}\left[z_{s}\right]$ on $m(S)$ by $\iota_{s} f(t)=f(s t)$ $\left[{ }_{s} f(t)=f(t s)\right]$. A mean on $S$ is a positive element of norm one in the dual $m(S)^{*}$ of $m(S)$. We say that $\mu \in m(S)^{*}$ is left [right] invariant if $\left.\mu\left(\ell_{s} f\right)=\mu(f)\left[\mu_{\iota_{s}} f\right)=\mu(f)\right]$ for each $f \in m(S)$ and $s \in S$. A semigroup is said to be left [right] amenable if it has a left [right] invariant mean, and we denote the set of left [right] invariant means on $S$ by $M \iota(S)\left[M_{2}(S)\right]$. When $S$ is both left and right amenable we say that $S$ is amenable. For a detailed account of the properties of left amenable semigroups, we refer to Day [2] and [3].

For any subset $A \subset S$, let $\chi_{A}$ denote its characteristic function, i.e., $\chi_{A}(s)=1$ if $s \in A$ and $\chi_{A}(s)=0$ if $s \notin A$. If $A$ is finite, we use $|A|$ to denote the cardinality of $A$. As usual, for each $s \in S$ we define $s A=\{s t \mid t \in A\}$ and $s^{-1} A=\{t \in S \mid s t \in A\}$.

We define a relation $R$ on any semigroup $S$ by $s R t$ for $s, t \in S$ if there exists $x \in S$ with $s x=t x$. If the intersection of finitely many right ideals of $S$ is always nonempty (as when $S$ is left amenable), then $R$ is an equivalence relation, and the set $S^{\prime}$ of equivalence classes is a right cancellative semigroup with the induced multiplication. More details are found in Granirer [7, p. 371]. When $S^{\prime}$ exists, we will refer to it as the right cancellative quotient semigroup of $S$.

Sorenson's conjecture that every right cancellative left amenable semigroup is left cancellative arose as a question of John Sorenson, who proved the weaker result that every right cancellative left 
measurable (definition in $\S 5$ ) semigroup is left cancellative in his thesis [16, p. 57] (see also [15]). The first discussion of this conjecture is found in a paper of Granirer [8, p. 108].

If this conjecture were true, then for any left amenable semigroup $S$, its right cancellative quotient semigroup $S^{\prime}$ would actually be cancellative and left amenable, and hence could be imbedded in an amenable group (Wilde and Witz [19, Cor. 3.6]). Thus in some sense the study of left amenable semigroups would essentially depend on the study of left amenable subsemigroups of groups. Further interest in the conjecture arose from the work of Argabright and Wilde on the strong Følner condition.

In [4] Følner introduced the following necessary and sufficient condition for a group $S$ to be left amenable:

(FC) For each finite subset $F$ of $S$ and $\varepsilon>0$, there exists a finite subset $A$ of $S$ such that $|s A \backslash A|<\varepsilon|A|$ for each $s \in F$.

In his thesis [5] Frey showed that every left amenable semigroup satisfies FC; however the converse is false since every finite semigroup satisfies FC, though not every finite semigroup is left amenable. A much simpler proof of Frey's result was given by Namioka [13] using the concept of strong amenability (see Day [3, §5]).

Continuing the search for a necessary and sufficient condition of this type for left amenability in semigroups, Argabright and Wilde [1] introduced the strong Følner condition (SFC) and showed that any semigroup satisfying SFC is left amenable.

(SFC) For each finite subset $F$ of $S$ and $\varepsilon>0$, there exists a finite subset $A$ of $S$ such that $|A| s A|<\varepsilon| A \mid$ for each $s \in F$.

Argabright and Wilde also showed that if Sorenson's conjecture were true, then every left amenable semigroup would satisfy SFC. However, the question of whether every left amenable semigroup must satisfy SFC remained open. We will refer to this question as the SFC problem. Further discussion on this problem and Sorenson's conjecture in relation to two conjectures of Granirer on extremely right amenable semigroups is found in Rajagopalan and Ramakrishnan [14].

In $\S 2$ we show that the SFC problem and Sorenson's conjecture are equivalent, in other words every left amenable semigroup satisfies SFC if and only if every right cancellative left amenable semigroup is left cancellative. This result follows directly from Theorem 2.2 which completely characterizes the semigroups which satisfy SFC as those left amenable semigroups whose right cancellative quotient semigroups are left cancellative. 
A counterexample to Sorenson's conjecture is constructed in $\S 3$ (3.5). In fact we exhibit a right cancellative amenable semigroup which neither is left cancellative, nor satisfies SFC. This shows that the answer to both Sorenson's conjecture and the SFC problem is still no, even if we replace left amenable by amenable. However, since all the counterxamples we have been able to find by our method are infinitely generated, the question is still open for finitely generated semigroups. The counterexample is obtained via an investigation of the semidirect product of semigroups in relation to amenability and cancellation properties. Several other examples and results on this topic are included in $\S 3$.

In $\S 4$, some properties of the class of semigroups satisfying SFC are described, following the work of Day ([2] and [3]) on left amenable semigroups.

Section 5 describes related results by Sorenson on left measurable semigroups, and concludes with a glance at semidirect products of left measurable semigroups.

2. Equivalence of the SFC problem to Sorenson's conjecture. After a simple lemma, we give a complete characterization of semigroups which satisfy $\mathrm{SFC}$ in Theorem 2.2. One direction of this theorem was proved by Argabright and Wilde [1]. From this characterization it will be obvious that Sorenson's conjecture and the SFC problem are equivalent (Corollary 2.3). Further results on the class of semigroups satisfying SFC are found in $\S 4$.

LEMMA 2.1. Let $S^{\prime}$ be the right cancellative quotient semigroup of a semigroup $S$. If $S^{\prime}$ is not left cancellative then there exist $r, s, t \in S$ with $r s=r t$ but $s x \neq t x$ for each $x \in S$.

Proof. Since $S^{\prime}$ is not left cancellative, there exist $r, s_{0}, t_{0} \in S$ with $r s_{0} y=r t_{0} y$ for some $y \in S$, but $s_{0} x \neq t_{0} x$ for each $x \in S$. Now let $s=s_{0} y$ and $t=t_{0} y$.

THEOREM 2.2. A semigroup $S$ satisfies SFC if and only if $S$ is left amenable and its right cancellative quotient semigroup $S^{\prime}$ is left cancellative.

Proof. Suppose $S$ is left amenable and $S^{\prime}$ is left cancellative. Then $S^{\prime}$ is left amenable since it is a homomorphic image of $S$, and hence must satisfy FC (Frey [5] or Namioka [13, Thm. 3.5]). Clearly for left cancellative semigroups, the conditions FC and SFC are equivalent, thus $S^{\prime}$ satisfies SFC. Argabright and Wilde showed that this implies that $S$ also satisfies SFC [1, Thm. 5]. This direction of 
the proof is contained in Argabright and Wilde [1].

Now suppose $S$ satisfies SFC. Then $S$ is left amenable [1, Thm. 1], so assume $S^{\prime}$ is not left cancellative. By Lemma 2.1 there exist $r, s, t \in S$ with $r s=r t$ but $s x \neq t x$ for each $x \in S$. By SFC we can find a finite subset $A \subset S$ such that $|A| r A|<1 / 5| A|| A,|s A|<1 / 5|A|$, and $|A| t A|<1 / 5| A \mid$. Now $\left|A \cap s^{-1} A\right| \geqq\left|s\left(A \cap s^{-1} A\right)\right|=|s A \cap A|>$ $4 / 5|A|$ since $|A| s A|<1 / 5| A \mid$, and similarly $\left|A \cap t^{-1} A\right|>4 / 5|A|$. Thus $\left|A \cap s^{-1} A \cap t^{-1} A\right|>3 / 5|A|$ and hence $|A|\left(s^{-1} A \cap t^{-1} A\right)|<2 / 5| A \mid$. This implies that $\left|s\left(A \backslash\left(s^{-1} A \cap t^{-1} A\right)\right)\right|<2 / 5|A|$, and since $|s A|>4 / 5|A|$ we must have $\left|A \cap s\left(t^{-1} A\right)\right|=\left|s\left(s^{-1} A \cap t^{-1} A\right)\right| \geqq\left|s\left(A \cap s^{-1} A \cap t^{-1} A\right)\right|>$ $2 / 5|A|$.

Let $B=\left(A \cap s\left(t^{-1} A\right)\right) \cup\left(A \cap t\left(s^{-1} A\right)\right)$. Clearly $|B|>2 / 5|A|$. We have $B \subset A$, and for each $y \in B$ there exists $y_{0} \in B \backslash\{y\}$ with $r y=r y_{0}$. To see this suppose $y \in A \cap s\left(t^{-1} A\right)$. Then $y=s x$ for some $x \in S$, where $t x \in A \cap t\left(s^{-1} A\right)$. Let $y_{0}=t x$. Clearly $y_{0} \in B, y_{0} \neq y$, and $r y=$ $r y_{0}$ by our choice of $r, s$, and $t$. A similar argument applies for $y \in A \cap t\left(s^{-1} A\right)$. Thus we must have $|r B| \leqq 1 / 2|B|$.

Now we see that $|r A \cap A| \leqq|r A| \leqq|r(A \backslash B)+| r B|\leqq| A \backslash B \mid+$ $1 / 2|B|=|A|-1 / 2|B|<4 / 5|A|$ since $|B|>2 / 5|A|$. This shows that $|A| r A|>1 / 5| A \mid$, but $A$ was chosen so that $|A| r A|<1 / 5| A \mid$. Thus $S^{\prime}$ must be left cancellative.

COROLlaRY 2.3. Every left amenable semigroup satisfies SFC if and only if every right cancellative left amenable semigroup is left cancellative.

Proof. This follows immediately from the theorem above, by noting that if $S$ is right cancellative and left amenable, then $S=S^{\prime}$.

3. The counterexample, semidirect products and amenability. For any semigroup $U$ we let $\operatorname{End}(U)$ denote the set of endomorphisms of $U$. We use the notation $\operatorname{Inj}(U), \operatorname{Sur}(U)$, and $\operatorname{Aut}(U)$ to denote the subsets of End $(U)$ consisting respectively of injective endomorphisms, surjective endomorphisms, and automorphisms.

Suppose that $U$ and $T$ are semigroups with a homomorphism $\rho: T \rightarrow$ End $(U)$. In general we will write $\rho_{a}$ for the endomorphism $\rho(a)$ for each $a \in T$. We define the semidirect product of $U$ by $T$ (with respect to $\rho$ ) as the semigroup $S$ of ordered pairs $(u, a)$ for $u \in U$ and $a \in T$, with the operation $(u, a)(v, b)=\left(u \rho_{a}(v), a b\right)$. It is easy to check that this operation is associative, hence $S$ is indeed a semigroup. We write $S=U \underset{\rho}{\times} T$, and refer to $U$ and $T$ as the factor semigroups.

This product is a natural generalization of the usual semidirect product of groups (see Gorenstein [6] for example). Its extension 
to semigroups has already been considered from various aspects (Hofmann and Mostert [11, D.4.1], Wells [17], among others), although not in the context of amenability as far as we know.

The counterexample to Sorenson's conjecture is constructed by taking the semidirect product of two cancellative amenable semigroups in such a way that the semidirect product is right cancellative, left amenable, but not left cancellative. In Lemmas 3.1 and 3.2, and Proposition 3.4 we assemble the information needed to show that the example given in 3.5 actually has the desired properties.

The rest of this section contains other results and examples which examine how amenability of the semidirect product is related to amenability of the factor semigroups.

LEMMA 3.1. If $U$ and $T$ are semigroups with a homomorphism $\rho: T \rightarrow$ End $(U)$ such that $\rho(T) \not \subset \operatorname{Inj}(U)$, then $S=U \underset{\rho}{\times} T$ is not left cancellative.

Proof. Suppose $a \in T$ and $u, v \in U$ with $u \neq v$ such that $\rho_{a}(u)=$ $\rho_{a}(v)$. Then $(u, a) \neq(v, a)$ but $(u, a)(u, a)=(u, a)(v, a)$.

Lemma 3.2. If $U$ and $T$ are right cancellative semigroups with a homomorphism $\rho: T \rightarrow \operatorname{End}(U)$, then $S=U \underset{\rho}{\times} T$ is right cancellative.

Proof. Suppose there exist $a, b, c \in T$ and $u, v, w \in U$ such that $(u, a)(w, c)=(v, b)(w, c)$. Then $a c=b c$ implies $a=b$, and $u \rho_{b}(w)=$ $u \rho_{a}(w)=v \rho_{b}(w)$ implies $u=v$. Thus $(u, a)=(v, b)$.

Given a homomorphism $\rho: T \rightarrow \operatorname{End}(U)$, for each $a \in T$ we define a linear operator $P_{a}$ on $m(U)$ by $P_{a} g(u)=g\left(\rho_{a}(u)\right)$ for $g \in m(U)$ and $u \in U$. Each $P_{a}$ induces a linear operator $P_{a}^{*}$ on $m(U)^{*}$ given by $P_{a}^{*} \psi(g)=\psi\left(P_{a} g\right)$ for $\psi \in m(U)^{*}$ and $g \in m(U)$.

Lemma 3.3. If $U$ and $T$ are left amenable semigroups with a homomorphism $\rho: T \rightarrow \operatorname{Sur}(U)$, then there exists $\phi \in M \ell(U)$ such that $P_{a}^{*} \phi=\phi$ for each $a \in T$.

Proof. For each $\psi \in M \iota(U)$ and $a \in T$ we have $P_{a}^{*} \psi \in M \iota(U)$ since $\rho_{a}$ is a homomorphism of $U$ onto $U$ (this follows from the proof that a homomorphic image of a left amenable semigroup is also left amenable, given in Day [2, p. 515]). Moreover, since $\rho: T \rightarrow \operatorname{Sur}(U)$ is a homomorphism, the map $a \rightarrow P_{a}^{*}$ is a representation of $T$ in the set of linear mappings on $M \iota(U)$. Since $M \iota(U)$ is compact and convex in the weak*-topology and since $T$ is left amenable, by the fixed 
point theorem (Day [3, Thm. 6.1]) there exists $\phi \in M \ell(U)$ with $P_{a}^{*} \phi=\phi$ for each $a \in T$.

Proposition 3.4. If $U$ and $T$ are left amenable semigroups with a homomorphism $\rho: T \rightarrow \operatorname{Sur}(U)$, then $S=U \times T$ is left amenable.

Proof. By the lemma above we can choose $\phi \in M \ell(U)$ such that $P_{a}^{*} \phi=\phi$ for each $a \in T$. For each $f \in m(S)$ define $\bar{f} \in m(T)$ by $\bar{f}(a)=$ $\phi\left(f_{a}\right)$, where $f_{a} \in m(U)$ is defined as $f_{a}(u)=f(u, a)$. Choose $\nu \in M \ell(T)$ and define $\mu \in m(S)^{*}$ by $\mu(f)=\nu(\bar{f})$. It is easy to see that $\mu$ is a mean, and moreover we claim that $\mu$ is left invariant. For $(v, b) \in$ $S$ and $a \in T$ we have $\left(\ell_{(v, b)} f\right)_{a}=P_{b} \ell_{v} f_{b a}$ since for any $u \in U,\left(\ell_{(v, b)}\right) f_{a}(u)=$ $\ell_{(v, b)} f(u, a)=f\left(v \rho_{b}(u), b a\right)=f_{b a}\left(v \rho_{b}(u)\right)=P_{b} \ell_{v} f_{b a}(u)$. Thus $\left(\overline{\ell_{(v, b)} f}\right)(a)=$ $\phi\left(P_{b} \iota_{v} f_{b a}\right)=\phi\left(f_{b a}\right)=\ell_{b} \bar{f}(a)$ since $P_{b}^{*} \phi=\phi$ and $\phi$ is left invariant on $U$. Hence $\mu\left(\ell_{(v, b)} f\right)=\nu\left(\overline{\ell_{(v, b)} f}\right)=\nu\left(\ell_{b} \bar{f}\right)=\nu(\bar{f})=\mu(f)$ since $\nu$ is left invariant on $T$. Thus $\mu \in M \ell(S)$, showing that $S$ is left amenable.

CounterexAmple 3.5. Let $U$ be the free abelian semigroup generated by the elements $\left\{u_{i} \mid i=0,1,2, \cdots\right\}$, and let $T$ be the infinite cyclic semigroup with generator $\{a\}$. We define $\rho: T \rightarrow \operatorname{Sur}(U)$ by $\rho_{a}\left(u_{i}\right)=u_{i-1}$ if $i \geqq 1$ and $\rho_{a}\left(u_{0}\right)=u_{0}$. Since $U$ and $T$ are cancellative abelian semigroups, by Lemma 3.2 and Proposition 3.4, the semigroup $S=U \times T$ is right cancellative and left amenable. However, since $\rho_{a}\left(u_{1}\right) \stackrel{\rho}{=} u_{0}=\rho_{a}\left(u_{0}\right)$ we have $\rho(T) \not \subset \operatorname{Inj}(U)$, and hence $S$ is not left cancellative.

Thus $S$ is indeed a counterexample to Sorenson's conjecture, and by Theorem 2.2, $S$ is also a left amenable semigroup which does not satisfy SFC. Corollary 3.11 will show that $S$ is actually amenable since $U$ and $T$ are amenable, which shows that Sorenson's conjecture is still false when left amenable is replaced by amenable. We have not been able to construct a finitely generated counterexample by the method above, which raises the question of whether Sorenson's conjecture holds for finitely generated semigroups.

REMARK 3.6. We give three examples of semidirect products of left amenable semigroups to illustrate the role that the condition $\rho: T \rightarrow \operatorname{Sur}(U)$ plays in Proposition 3.4. The first example shows that the condition is not necessary to ensure left amenability of $U \times T$, but examples (ii) and (iii) show that some condition is needed since neither $\rho: T \rightarrow \operatorname{End}(U)$ nor $\rho: T \rightarrow \operatorname{Inj}(U)$ is sufficient.

(i) Let $U$ be any semigroup with at least two elements, including a zero element 0 , and let $T$ be the trivial semigroup $\{1\}$. Define $\rho_{1} \in \operatorname{End}(U)$ by $\rho_{1}(u)=0$ for each $u \in U$. Then for any $u, v \in U$. 
we have $(u, 1)(v, 1)=(0,1)$, thus $U \times T$ is left amenable, but since $U$ has at least two elements $\left.\rho_{1} \notin \operatorname{Sur} \stackrel{\rho}{(} U\right)$.

(ii) Let $T$ be any amenable semigroup, and $U$ any amenable semigroup of at least two elements and containing an identity $e$. We define $\rho: T \rightarrow$ End $(U)$ by $\rho_{a}(u)=e$ for each $a \in T$ and $u \in U$. We have $(u, a)(v, b)=(u e, a b)=(u, a b)$ for any $(u, a),(v, b) \in U \times T$. Thus if $u, v \in U$ with $u \neq v$, we see that $(u, a)(U \times T) \cap(v, a)\left(U^{\rho} \times T\right)=\phi$, which shows that $U \times T$ is not left amenable.

(iii) Let $U$ be the nonnegative integers under addition, and let $T$ be the infinite cyclic semigroup with generator $\{a\}$. Define $\rho: T \rightarrow$ $\operatorname{Inj}(U)$ by $\rho_{\alpha}(u)=2 u$ for each $u \in U$. Now we see that

$$
(0, a)(U \underset{\rho}{\times} T)=\left\{\left(u, a^{j}\right) \mid u \quad \text { even }, \quad j=2,3, \cdots\right\}
$$

and

$$
(1, a)(U \underset{\rho}{\times} T)=\left\{\left(u, a^{j}\right) \mid u \quad \text { odd }, \quad j=2,3, \cdots\right\} .
$$

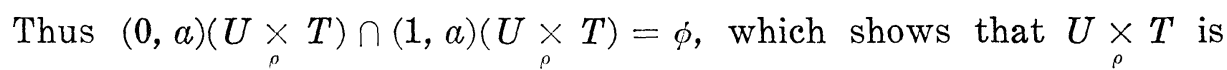
not left amenable.

REMARK 3.7. Suppose $S=U \times T$. Then we may add a twosided identity to either $U$ or $T$ (or both) and extend the homomorphism $\rho$ in such a way that $S$ contains a two-sided ideal of the new semidirect product obtained. Let $U^{0}$ be the semigroup obtained by adding a two-sided identity $e$ to $U$. Then by defining $\rho^{0}: T \rightarrow \operatorname{End}\left(U^{0}\right)$ by $\rho_{a}^{0}(u)=\rho_{a}(u)$ for $u \in U$ and $\rho_{a}^{n}(e)=e$ for each $a \in T$, we see that $\left(U^{0} \times \rho_{0}^{0} T\right)(u, a)\left(U^{0} \underset{\rho^{0}}{\times} T\right) \subset S$ for each $(u, a) \in S$. Similarly if $T^{0}$ is the semigroup obtained by adding a two-sided identity 1 to $T$, we define $\rho_{a}^{0}=\rho_{a}$ for each $a \in T$ and $\rho_{1}^{n}=$ identity homomorphism on $U$. Once again we have $\left(U \underset{\sigma_{0}}{\times} T^{0}\right)(u, a)\left(U \underset{\rho_{0}}{\times} T^{0}\right) \subset S$ for each $(u, a) \in S$.

This remark will be useful in the propositions which follow, since it is well-known (Mitchell [12, Thm. 9] for example) that if $A$ is a subsemigroup of $B$ containing a two-sided ideal of $B$, then $A$ is left [right] amenable if and only if $B$ is left [right] amenable.

Proposition 3.8. If $S=U \times T$ is left amenable, then $U$ and $T$ are left amenable.

Proof. The map $\sigma: S \rightarrow T$ defined by $\sigma(u, a)=a$ is a homomorphism from $S$ onto $T$, which shows that $T$ is left amenable.

To show that $U$ is left amenable, by Remark 3.7 we may assume 
without loss of generality that $T$ has an identity 1 and that $\rho_{1}$ is the identity map on $U$. For each $f \in m(U)$ define $f^{\sim} \in m(S)$ by $f^{\sim}(u, a)=$ $f(u)$. Notice that for each $v \in U$ we have $\left(\ell_{v} f\right)^{\sim}=\iota_{(v, 1)} f^{\sim}$ since $\left(\iota_{v} f\right) \sim(u, a)=\iota_{v} f(u)=f(v u)=f \sim(v u, a)=\iota_{(v, 1)} f \sim(u, a)$. Choosing $\nu \in$ $M \iota(S)$, we define $\mu \in m(U)^{*}$ by $\mu(f)=\nu\left(f^{\sim}\right)$. Actually $\mu \in M \iota(U)$ since $\mu$ is clearly a mean, and $\mu\left(\ell_{v} f\right)=\nu\left(\left(\ell_{v} f\right)^{\sim}\right)=\nu\left(\ell_{(v, 1)} f^{\sim}\right)=\nu\left(f^{\sim}\right)=$ $\mu(f)$.

REMARK 3.9. The left invariant mean $\mu$ on $U$ constructed in the above proof has the property $P_{a}^{*} \mu=\mu$ for each $a \in T$. To see this, choose any $u \in U$ and notice that for $f \in m(U)$ we have $\left(\ell_{u} P_{a} f\right)^{\sim}=$ $\ell_{\left(\rho_{a}(u), a\right)} f^{\sim}$, since if $(v, b) \in U \times T$ we have $\left(\ell_{u} P_{a} f\right) \sim(v, b)=\ell_{k} P_{a} f(v)=$ $P_{a} f(u v)=f\left(\rho_{a}(u) \rho_{a}(v)\right)$, and $\ell_{\left(\rho_{a}(u), a\right)} f^{\sim}(v, b)=f^{\sim}\left(\left(\rho_{a}(u), a\right)(v, b)\right)=$ $f^{\sim}\left(\rho_{a}(u) \rho_{a}(v), a b\right)=f\left(\rho_{a}(u) \rho_{a}(v)\right)$. Thus $\mu\left(P_{a} f\right)=\mu\left(\ell_{u} P_{a} f\right)=\nu\left(\left(\ell_{u} P_{a} f\right)^{\sim}\right)=$ $\nu\left(\ell_{\left(\rho_{a}(u), a\right)} f^{\sim}\right)=\nu\left(f^{\sim}\right)=\mu(f)$.

Proposition 3.10. If $U$ and $T$ are right amenable semigroups with a homomorphism $\rho: T \rightarrow$ End $(U)$, then $S=U \times T$ is right amenable.

Proof. Choose $\phi \in M_{z}(U)$ and $\nu \in M_{z}(T)$. For each $f \in m(S)$ we define $\bar{f} \in m(T)$ by the formula $\bar{f}(a)=\phi\left(f_{a}\right)$, where $f_{a} \in m(U)$ is defined by $f_{a}(u)=f(u, a)$ for each $a \in T$ and $u \in U$. Now we define $\mu \in m(S)^{*}$ by $\mu(f)=\nu(\bar{f})$ for each $f \in m(S)$. It is easy to check that for $(v, b) \in$ $S$ we have $\left(z_{(v, b)} f\right)_{a}=z_{\rho_{a}(v)} f_{a b}$, and hence $\overline{z_{(v, b)} f}=z_{b} \bar{f}$. Thus $\mu\left(z_{(v, b)} f\right)=$ $\nu\left(\overline{z_{(v, b)} f}\right)=\nu\left(z_{b} \bar{f}\right)=\mu(f)$. Since $\mu$ is also a mean on $S$, we see that $S$ is right amenable.

CoRollary 3.11. If $U$ and $T$ are amenable semigroups with $a$ homomorphism $\rho: T \rightarrow \operatorname{Sur}(U)$, then $S=U \times T$ is amenable.

Proof. This is immediate from Propositions 3.4 and 3.10 .

Proposition 3.12. If $S=U \times T$ is right amenable then $T$ is right amenable, and if in addition $\rho: T \rightarrow \operatorname{Aut}(U)$ then $U$ is right amenable also.

Proof. As before $T$ is a homomorphic image of $S$ and hence right amenable. Now suppose $\rho: T \rightarrow \operatorname{Aut}(U)$. By Remark 3.7 we may assume that $T$ has an identity 1 and that $\rho_{1}$ is the identity map on $U$. For $f \in m(U)$ we define $f^{\vee} \in m(S)$ by $f^{\vee}(u, a)=f\left(\rho_{a}^{-1}(u)\right)$, where $\rho_{a}^{-1}$ denotes the inverse automorphism to $\rho_{a}$. Now for each $v \in U$, we have $\left(z_{v} f\right)^{\vee}(u, a)=f\left(\rho_{a}^{-1}(u) v\right)=f\left(\rho_{a}^{-1}\left(u \rho_{a}(v)\right)\right)=f^{\vee}\left(u \rho_{a}(v), a\right)={ }_{z_{(v, 1)}} f^{\vee}(u, a)$. 
Choosing $\nu \in M_{z}(S)$ we define $\mu \in m(U)^{*}$ by $\mu(f)=\nu\left(f^{\vee}\right)$. It is easy to see that $\mu$ is a right invariant mean on $U$.

Corollary 3.13. If $S=U \times T$ is amenable then $T$ is amenable, and if in addition $\rho: T \rightarrow \operatorname{Aut}(\stackrel{\rho}{U})$, then $U$ is also amenable.

Proof. This is immediate from Propositions 3.8 and 3.12.

REMARK 3.14. We give two examples to show that the condition $\rho: T \rightarrow \operatorname{Aut}(U)$ in Proposition 3.12 cannot be replaced by either $\rho: T \rightarrow$ $\operatorname{Sur}(U)$ or $\rho: T \rightarrow \operatorname{Inj}(U)$. Example (iii) shows that we cannot replace $\rho: T \rightarrow \operatorname{Aut}(U)$ in Corollary 3.13 by $\rho: T \rightarrow \operatorname{End}(U)$.

(i) We construct a right amenable semigroup $S=U \times T$ where $\rho: T \rightarrow \operatorname{Sur}(U)$, but $U$ is not right amenable. Let $U$ be the free semigroup on the generators $\left\{u_{i} \mid i=0,1,2, \cdots\right\}$, and let $T$ be the infinite cyclic semigroup generated by $\{a\}$. We define $\rho: T \rightarrow \operatorname{Sur}(U)$ by $\rho_{a}\left(u_{i}\right)=u_{i-1}$ for $i \geqq 1$ and $\rho_{a}\left(u_{0}\right)=u_{0}$. Since $U$ is clearly not right amenable, all that remains to be shown is that $S$ is right amenable. Actually we show that $S$ satisfies the "right-sided" version of the strong Følner condition: $\left(\mathrm{SFC}_{z}\right)$ For any finite subset $F \subset S$ and $\varepsilon>0$, there exists a finite subset $A \subset S$ with $|A \backslash A s|<\varepsilon|A|$ for each $s \in F$.

Thus suppose $F$ is a finite subset and $\varepsilon>0$. Then there exists an integer $N$ such that

$$
\left\{\left(\rho_{a^{N}}(u), a^{i}\right) \mid\left(u, a^{i}\right) \in F\right\} \subset\left\{\left(u_{0}^{j}, a^{k}\right) \mid 1 \leqq j, k \leqq N\right\} .
$$

Choose $M$ so that $2 N / M<\varepsilon$, and let $A=\left\{\left(u_{0}^{m}, a^{n+N}\right) \mid 1 \leqq m, n \leqq M\right\}$. Then for each $\left(u, a^{i}\right) \in F$ it is easy to check that

$$
\left\{\left(u_{0}^{m}, a^{n+N}\right) \mid N+1 \leqq m, n \leqq M\right\} \subset A\left(u, a^{i}\right),
$$

which shows that $\left|A \backslash A\left(u, a^{i}\right)\right|<2 N M=(2 N / M) M^{2}<\varepsilon|A|$.

(ii) We construct a right amenable semigroup $S=U \times T$ where $\rho: T \rightarrow \operatorname{Inj}(U)$, but $U$ is not right amenable. Let $U$ be the semigroup generated by the elements $\left\{u_{i}, v_{i} \mid i=1,2, \cdots\right\}$ with the relations $u_{i} v_{j}=v_{j} u_{i}=u_{i} u_{j}=u_{j} u_{i}=u_{i}$ if $i<j$, and $u_{i} v_{j}=v_{j} u_{i}=v_{i} v_{j}=v_{j} v_{i}=$ $v_{j}$ if $j<i$. Notice that for each $i$, the semigroup generated by $\left\{u_{i}, v_{i}\right\}$ is the free semigroup on two generators, and hence $U u_{1} \cap$ $U v_{1}=\phi$. This shows that $U$ is not right amenable.

Let $T$ be the infinite cyclic semigroup generated by $\{a\}$, and define $\rho: T \rightarrow \operatorname{Inj}(U)$ by $\rho_{a}\left(u_{i}\right)=u_{i+1}$ and $\rho_{a}\left(v_{i}\right)=v_{i+1}$. To see that $S=U \times T$ is right amenable we show that $S$ satisfies $\mathrm{SFC}_{2}$ as in example (i) above. Suppose $F \subset S$ is a finite subset and $\varepsilon>0$. Let 
$N=\sup \left\{n \mid\left(u, a^{n}\right) \in F\right.$ for some $\left.u \in U\right\}$, and choose $M$ so that $N / M<$ ع. Letting $A=\left\{\left(u_{1}, a^{k}\right) \mid 1 \leqq k \leqq M\right\}$, we see that for each $\left(u, a^{n}\right) \in F$ we have $A\left(u, a^{n}\right)=\left\{\left(u_{1}, a^{k+n}\right) \mid 1 \leqq k \leqq M\right\}$ since $u_{1} \rho_{a}(u)=u_{1}$ for all $u \in U$. Also since $n \leqq N$ we have $|A| A\left(u, a^{n}\right)|\leqq N=(N / M)| A \mid<$ $\varepsilon|A|$.

(iii) We construct an amenable semigroup $S=U \times T$ with $\rho: T \rightarrow \operatorname{End}(U)$, such that $U$ is not right amenable, hence not amenable. Let $U=\{u, v\}$ where $u^{2}=v u=u$ and $v^{2}=u v=v$. We choose $T$ to be the trivial semigroup $\{1\}$, and define $\rho: T \rightarrow \operatorname{End}(U)$ by $\rho_{1}(u)=\rho_{1}(v)=u$. Clearly $U$ is not right amenable since $U u \cap U v=\phi$. However $(u, 1)(v, 1)=(v, 1)(u, 1)$ which shows that $S$ is abelian and hence amenable.

4. Semigroups satisfying SFC. From the counterexample constructed in 3.5 we know that the class of semigroups satisfying SFC is a proper subset of the class of left amenable semigroups. In this section we examine some of the properties of this class, generally following the line of results established for left amenable semigroups (see Day [2] and [3]).

REMARK 4.1. By Lemma 2.1 and Theorem 2.2, in order to show that a particular left amenable semigroup $S$ satisfies SFC, we need only show that whenever $r s=r t$ for some $r, s, t \in S$, there exists $x \in S$ with $s x=t x$.

PROPOSITION 4.2. If $S$ is a semigroup satisfying SFC with a subsemigroup $T$ such that $\mu\left(\chi_{T}\right)>0$ for some $\mu \in M \ell(S)$, then $T$ also satisfies SFC.

Proof. $T$ is left amenable since $\mu\left(\chi_{T}\right)>0$ (Day [2]). Suppose $a, b, c \in T$ with $a b=a c$. Since $S$ satisfies SFC there exists $x \in S$ with $b x=c x$. Since $\mu\left(\chi_{T}\right)>0$ and $\mu\left(\chi_{x S}\right)=1, T \cap x S$ is nonempty. Hence we can pick $d \in T \cap x S$, and we have $b d=c d$. This shows that $T$ satisfies SFC by Remark 4.1.

It is not true, however, that every left amenable subsemigroup of a semigroup satisfying SFC must satisfy SFC. For example, consider a semigroup obtained by adding a two-sided zero to another semigroup which is left amenable but does not satisfy SFC.

A subset $T \subset S$ is said to be left thick if for every finite subset $F \subset S$ there exists $a \in T$ such that $F a \subset T$. Clearly any subset containing a left ideal of $S$ is left thick. Mitchell [12, Thm. 7] showed that a subset $T$ of a left amenable semigroup $S$ is left thick if and only if $\mu\left(\chi_{T}\right)=1$ for some $\mu \in M \iota(S)$. 
Proposition 4.3. If $T$ is a left thick subset of $S$ such that $T$ satisfies $\mathrm{SFC}$, then $S$ satisfies SFC.

Proof Let $F$ be a finite subset of $S$ and $\varepsilon>0$. Choose $\delta>0$ such that $2 \delta /(1-\delta)<\varepsilon$. Since $T$ is left thick there exists $a \in T$ such that $F a \subset T$. Choose a finite subset $B \subset T$ such that $|B \backslash a B|<$ $\delta|B|$ and $|B \backslash s a B|<\delta|B|$ for each $s \in F$. Now we see that

$$
|a B| s a B|\leqq 2 \delta| B\left|<2 \delta\left[\frac{|a B|}{(1-\delta)|B|}\right]\right| B|<\varepsilon| a B \mid .
$$

This proposition extends a result of Rajagopalan and Ramakrishnan [14, Thm. 22]. The next two results are stated without proof since their verification is routine.

Proposition 4.4. A finite direct product of semigroups which satisfy SFC also satisfies SFC.

Proposition 4.5. A directed union of semigroups which satisfy SFC also satisfies SFC.

Proposition 4.6. If $U$ and $T$ are semigroups which satisfy SFC with a homomorphism $\rho: T \rightarrow$ Aut $(U)$, then $S=U \times \underset{\rho}{\times} T$ satisfies SFC.

Proof. $S$ is left amenable by Proposition 3.4. Suppose there exist $(u, a),(v, b)$, and $(w, c) \in S=U \times T$ with $(u, a)(v, b)=(u, a)(w, c)$. Then $u \rho_{a}(v)=u \rho_{a}(w)$ implies $\rho_{a}^{-1}(u) v^{\rho}=\rho_{a}^{-1}(u) w$, and hence there exists $y \in U$ with $v y=w y$. Also $a b=a c$ implies there exists $d \in T$ with $b d=c d$. Since $\rho_{d}$ is surjective and $\rho_{b} \rho_{d}=\rho_{c} \rho_{d}$ we must have $\rho_{b}=\rho_{c}$. Thus we see that $(v, b)\left(\rho_{b}^{-1}(y), d\right)=(v y, b d)=(w y, c d)=(w, c)\left(\rho_{c}^{-1}(y), d\right)=$ $(w, c)\left(\rho_{b}^{-1}(y), d\right)$. By Remark 4.1 we have shown that $S$ satisfies SFC.

Examples 3.5 and 3.6 (iii) show respectively that the condition $\rho: T \rightarrow \operatorname{Aut}(U)$ in the proposition above cannot be replaced by either $\rho: T \rightarrow \operatorname{Sur}(U)$ or $\rho: T \rightarrow \operatorname{Inj}(U)$.

Proposition 4.7. If $S=U \times T$ satisfies $\mathrm{SFC}$, then $U$ and $T$ also satisfy SFC.

Proof. $U$ and $T$ are left amenable by Proposition 3.8. Moreover, Remark 3.7 combined with Propositions 4.2 and 4.3 shows that we may assume that $T$ has an identity 1 and that $\rho_{1}$ is the identity map on $U$. Suppose $u v=u w$ for $u, v, w \in U$. Then $(u, 1)(v, 1)=(u, 1)(w, 1)$, 
hence there exists $(y, a) \in S$ with $(v, 1)(y, a)=(w, 1)(y, a)$. From this we see $v y=w y$, which shows that $U$ satisfies SFC.

Now suppose $a b=a c$ for $a, b, c \in T$. Then $(u, a)(u, b)=(u, a)(u, c)$ for any $u \in U$, thus there exists $(v, d) \in S$ with $(u, b)(v, d)=(u, c)(v, d)$. Hence we have $b d=c d$, showing that $T$ satisfies SFC.

The question of whether homomorphic images of semigroups satisfying SFC also satisfy SFC is still open. An answer to this would be particularly interesting in view of the corresponding results for left amenable semigroups (yes, Day [2]) and left measurable semigroups (no, Sorenson [16]).

5. Left measurable semigroups. For any semigroup $S$ it is easy to see that a mean $\mu \in m(S)^{*}$ is left invariant if and only if $\mu\left(\chi_{s-1}\right)=\mu\left(\chi_{A}\right)$ for each $A \subset S$ and $s \in S$. We say that a mean $\mu \in$ $m(S)^{*}$ is left reversible invariant if $\mu\left(\chi_{s A}\right)=\mu\left(\chi_{A}\right)$ for each $A \subset S$ and $s \in S$, and denote the set of left reversible invariant means on $S$ by $R M \iota(S)$. If a semigroup $S$ has a left reversible invariant mean we say that $S$ is left measurable. This term arises from the obvious one-to-one correspondence between $R M \iota(S)$ and the set of left measures on $S$, i.e., the set of finitely additive measures $\lambda$ on $S$ such that $\lambda(S)=1$ and $\lambda(s A)=\lambda(A)$ for each $s \in S$ and $A \subset S$. Clearly every left measurable semigroup is left amenable since any left reversible invariant mean is left invariant, and also for left cancellative semigroups the conditions are equivalent. The terms right reversible invariant and right measurable are defined analogously.

Sorenson investigated the properties of left measurable semigroups in his thesis [16]. In particular he showed that every left measurable right cancellative semigroup is left cancellative [16, 3.1.7]. The proof that follows in Theorem 5.1 is not the one that Sorenson gave, although he noticed that this type of proof was possible [16, remarks on p. 57].

THEOREM 5.1. If $S$ is a left measurable semigroup, then its right cancellative quotient semigroup $S^{\prime}$ is left cancellative.

Proof. Suppose $S^{\prime}$ is not left cancellative. Then by Lemma 2.1 there exist $r, s, t \in S$ such that $r s=r t$ but $s x \neq t x$ for each $x \in S$. Let $\mathscr{B}=\{A \subset S \mid s A \cap t A=\phi\}$. Then $\mathscr{B} \neq \phi$ since $\{x\} \in \mathscr{B}$ for each $x \in S$. If we partially order $\mathscr{B}$ by inclusion, it is easy to see that $\mathscr{B}$ is chainable, thus by Zorn's lemma let $A$ be a maximal element in $\mathscr{B}$. For each $x \in S \backslash A$ we have either $s x \in t A$ or $t x \in s A$ since $s x \neq$ $t x$ and $A$ is maximal. Thus we may write $S=A \cup S_{1} \cup S_{2}$ where $s S_{1} \subset t A$ and $t S_{2} \subset s A$. If $\mu \in R M \iota(S)$, we must have $\mu\left(\chi_{A}\right)=\mu\left(\chi_{t A}\right) \geqq$ 
$\mu\left(\chi_{s S_{1}}\right)=\mu\left(\chi_{S_{1}}\right)$ and similarly $\mu\left(\chi_{A}\right) \geqq \mu\left(\chi_{S_{2}}\right)$, which shows that $\mu\left(\chi_{A}\right) \geqq$ 1/3. However, $r s=r t$ implies that $\mu\left(\chi_{A}\right)=\mu\left(\chi_{r s A}\right)=\mu\left(\chi_{(s A \cup t A)}\right)=$ $2 \mu\left(\chi_{A}\right)$, and hence $\mu\left(\chi_{A}\right)=0$. By this contradiction we see that $S^{\prime}$ must be left cancellative.

CoROLlary 5.2. If $S$ is left measurable and right cancellative, then $S$ is left cancellative.

Proof. If $S$ is right cancellative then $S=S^{\prime}$.

COROLlaRY 5.3. Every left measurable semigroup satisfies SFC.

Proof. This follows immediately from Theorems 2.2 and 5.1.

It is not true that every semigroup which satisfies $\mathrm{SFC}$ is left measurable, since any semigroup with a zero element obviously satisfies SFC, but cannot be left measurable if it has more than one element.

We now state some of the properties of left measurable semigroups obtained by Sorenson.

(i) The homomorphic image of a left measurable semigroup is not necessarily left measurable [16, Example 1, §3.1].

(ii) A left ideal of a left measurable semigroup need not be left measurable [16, Example 2, §3.1].

(iii) A right ideal of a left measurable semigroup is left measurable [16, 3.1.2].

(iv) A finite direct product of left measurable semigroups is left measurable [16, 3.1.4].

(v) A directed union of left measurable semigroups is left measurable $[16,3.1 .5]$.

The next lemma is useful in proving Propositions 5.5 and 5.6, which look at the semidirect product in relation to left measurability, following the pattern of results established in $\S 3$ and $\S 4$ for amenable semigroups and semigroups satisfying $\mathrm{SFC}$ respectively.

LeMma 5.4. A mean $\mu$ is left reversible invariant on a semigroup $S$ if and only if $\mu$ is left invariant and $\mu\left(\chi_{Z_{s}}\right)=1$ for each $s \in S$, where $Z_{s}=\left\{t \in S \mid s^{-1}\{s t\}=\{t\}\right\}$.

Proof. Suppose $\mu \in R M /(S)$ and $s \in S$. It is clear from the definition of $Z_{s}$, that by using a Zorn's lemma argument we may write $S \backslash Z_{s}=A_{1} \cup A_{2}$, where $A_{1} \cap A_{2}=\phi$ and $s A_{1}=s A_{2}=s\left(S \backslash Z_{s}\right)$. This implies that $\mu\left(\chi_{A_{1}}\right)=\mu\left(\chi_{A_{2}}\right)=\mu\left(\chi_{\left(S \backslash Z_{s}\right)}\right.$ and also that $\mu\left(\chi_{\left(s \backslash Z_{s}\right)}\right)=$ 
$\mu\left(\chi_{A_{1}}\right)+\mu\left(\chi_{A_{2}}\right)$. From this we see that $\mu\left(\chi_{\left(S \backslash Z_{s}\right)}\right)=0$, and hence $\mu\left(\chi_{z_{s}}\right)=1$.

Now suppose that $\mu \in M \iota(S)$ and $\mu\left(\chi_{z_{s}}\right)=1$ for each $s \in S$. For any set $A \subset S$ we have $s^{-1}(s A) \backslash A \subset S \backslash Z_{s}$, and hence $\left.\mu_{\left(\chi_{\left(s^{-1}(s A) \backslash A\right.}\right)}\right)=0$. Thus $\mu\left(\chi_{s A}\right)=\mu\left(\chi_{s^{-1}(s A)}\right)=\mu\left(\chi_{A}\right)+\mu\left(\chi_{\left(s^{-1}(s A) \backslash A\right.}\right)=\mu\left(\chi_{A}\right)$, which shows that $\mu$ is left reversible invariant on $S$.

The first part of this proof is given by Sorenson in [16, 2.3.2].

Notice that for a left amenable semigroup $S$ to be left measurable it must be "almost" left cancellative, in the sense that $Z_{s}$ must be left thick in $S$ for each $s \in S$.

Proposition 5.5. If $U$ and $T$ are left measurable semigroups with a homomorphism $\rho: T \rightarrow$ Aut $(U)$, then $S=U \times T$ is left measurable.

Proof. Recall that in $\S 3$ we defined $P_{a}: m(U) \rightarrow m(U)$ by $P_{a} f(u)=$ $f\left(\rho_{a}(u)\right.$ ), which induced a linear operator $P_{a}^{*}$ on $m(U)^{*}$. Since $\rho_{a} \in$ Aut $(U)$, it is straightforward to show that $P_{a}^{*}$ maps $R M \ell(U)$ onto itself, and since $R M \iota(U)$ is compact and convex with respect to the weak*-topology (Sorenson $[16,1.1 .10]$ ), once again we apply the fixed point theorem (Day [3, Thm. 6.1]) to obtain a left reversible invariant mean $\phi$ on $U$ such that $P_{a}^{*} \phi=\phi$ for each $a \in T$.

For each $f \in m(S)$ define $f_{a} \in m(U)$ by $f_{a}(u)=f(u, a)$, and define $\bar{f} \in m(T)$ by $\bar{f}(a)=\phi\left(f_{a}\right)$. Choosing $\nu \in R M \iota(T)$, we define $\mu \in m(S)^{*}$ by $\mu(f)=\nu(\bar{f})$. From the proof of Proposition 3.4 we know that $\mu \in M \iota(S)$, thus by Lemma 5.4 we need only show that $\mu\left(\chi_{z_{(u, a)}}\right)=1$ for each $(u, a) \in S$. After noting that $Z_{(u, a)}=\left\{(v, b) \mid v \in Z_{\rho_{a}^{-1}(u)}\right.$ and $\left.b \in Z_{a}\right\}$, we see that $\left(\chi_{Z_{(u, a)}}\right)_{b}=\chi_{z_{\rho_{a}^{-1}(u)}}$ if $b \in Z_{a}$, and $\left(\chi_{z_{(u, a)}}\right)_{b}=0$ if $b \notin Z_{a}$. Since $\phi\left(\chi_{z_{a}^{-1}(u)}\right)=1$ by Lemma 5.4, we see that $\overline{\chi_{z_{(u, a)}}}=\chi_{z_{a}}$. Now we have $\mu\left(\chi_{z_{(u, a)}}\right)=\nu\left(\chi_{z_{a}}\right)=1$ by Lemma 5.4, as desired.

It is not possible to replace the condition $\rho: T \rightarrow \operatorname{Aut}(U)$ int his proposition by either $\rho: T \rightarrow \operatorname{Sur}(U)$ or $\rho: T \rightarrow \operatorname{Inj}(U)$, as is shown by the Examples 3.5 and 3.6(iii) respectively.

Proposition 5.6. If $S=U \times T$ is left measurable, then $U$ and $T$ are left measurable.

Proof. Let $\nu \in R M \ell(S)$. To see that $U$ is left measurable, we define $\mu \in m(U)^{*}$ as in Proposition 3.8 by $\mu(f)=\nu\left(f^{\sim}\right)$, where $f^{\sim} \in m(S)$ is defined by $f \sim(u, a)=f(u)$ for each $f \in m(U)$. In the proof of Proposition 3.8 we saw that $\mu \in M \iota(U)$, hence it suffices to show that $\mu\left(\chi_{z_{u}}\right)=1$ for each $u \in U$. First consider the set $Z_{\left(\rho_{a}(u), a\right)}$ for some 
fixed $a \in T$. Now we have $\left(\chi_{z_{u}}\right) \geqq \chi_{z_{\left\langle\rho_{a}(u), a\right)}}$, since if $v \notin Z_{u}$ there exists $w \neq v$ with $u v=u w$. Then for any $b \in T$ we have $\left(\rho_{a}(u), a\right)(v, b)=$ $\left(\rho_{a}(u), a\right)(w, b)$ which shows that $(v, b) \notin Z_{\left(\rho_{a}(u), a\right)}$ for each $b \in T$. Now we have $1 \geqq \mu\left(\chi_{z_{u}}\right)=\nu\left(\left(\chi_{z_{u}}\right)\right) \geqq \nu\left(\chi_{z_{\left(\rho_{a}(u), a\right)}}\right)=1$ by Lemma 5.4 .

Similarly, to see that $T$ is left measurable we define $\psi \in m(T)^{*}$ by $\psi(g)=\nu\left(g^{\wedge}\right)$, where $g^{\wedge} \in m(S)$ defined by $g^{\wedge}(u, a)=g(a)$ for each $g \in m(T)$. After checking that $\psi$ is a left invariant mean, we see that $\psi\left(\chi_{z_{a}}\right)=1$ for each $a \in T$, since $\left(\chi_{z_{a}}\right)^{\wedge} \geqq \chi_{z_{(u, a)}}$ for any $u \in U$.

Combining the appropriate version of Lemma 5.4 for right reversible invariant means with Propositions 3.10 and 3.12, analogous arguments yield the following results:

Proposition 5.7. If $U$ and $T$ are right measurable semigroups with a homomorphism $\rho: T \rightarrow$ End $(U)$, then $S=U \times T$ is right measurable.

Proposition 5.8. If $S=U \times T$ is right measurable and $\rho: T \rightarrow$ Aut $(U)$, then $U$ and $T$ are right measurable.

\section{REFERENCES}

1. L. N. Argabright and C. O. Wilde, Semigroups satisfying a strong Folner condition, Proc. Amer. Math. Soc., 18 (1967), 587-591.

2. M. M. Day, Amenable semigroups, Illinois J. Math., 1 (1957), 509-544.

3. - Semigroups and Amenability, Semigroups, K. Folley, Ed., Academic Press, New York, 1969, 5-53.

4. E. Folner, On groups with full Banach mean values, Math. Scand., 3 (1955), 243254.

5. A. H. Frey, Jr., Studies on amenable semigroups, Thesis, University of Washington, 1960.

6. D. Gorenstein, Finite Groups, Harper and Row, New York, 1968.

7. E. Granirer, $A$ theorem on amenable semigroups, Trans. Amer. Math. Soc., 111 (1964), 367-379.

8. E. Granirer, Extremely amenable semigroups II, Math. Scand., 20 (1967), 93-113.

9. F. P. Greenleaf, Invariant means on topological groups and their applications, Van Nostrand Math. Studies, no. 16, Van Nostrand Reinhold, New York, 1969.

10. E. Hewitt and K. Ross, Abstract Harmonic Analysis, Vol. 1, Springer, Berlin, 1963, 11. K. H. Hofmann and P. S. Mostert, Elements of Compact Semigroups, C. E. Merrill Books, Columbus, Ohio, 1966.

12. T. Mitchell, Constant functions and left invariant means on semigroups, Trans. Amer. Math. Soc., 119 (1965), 244-261.

13. I. Namioka, Folner's conditions for amenable semigroups, Math. Scand., 15 (1964), 18-28.

14. M. Rajagopalan and P. V. Ramakrishnan, On a conjecture of Granirer and strong Folner condition, J. Indian Math. Soc., 37 (1973), 85-92.

15. J. R. Sorenson, Left-amenable semigroups and cancellation, Notices of Amer. Math. Soc., 11, No. 7, (1964), 763.

16. - Existence of measures that are invariant under a semigroup of transformations, Thesis, Purdue University, 1966. 
17. C. Wells, Some applications of the wreath product construction, Amer. Math. Monthly, 83 (1976), 317-338.

18. C. O. Wilde and L. N. Argabright, Invariant means and factor-semigroups, Proc. Amer. Math. Soc., 18 (1967), 226-228.

19. C. O. Wilde and K. Witz, Invariant means and Stone-Čech compactification, Pacific J. Math., 21 (1967), 577-586.

Received January 3, 1977 and in revised form April 11, 1977. I would like to thank Anthony T. Lau, whose observations on my original counterexample to Sorenson's conjecture lead me to the investigation of the semidirect product and helped to shorten the proof of Proposition 3.4, along with many other useful suggestions and comments. This paper will form part of my thesis under his supervision. I would also like to thank the National Research Council of Canada for their support in the form of a postgraduate scholarship.

The University of Alberta

Edmonton, Alberta, Canada T6G 2G1 


\section{PACIFIC JOURNAL OF MATHEMATICS}

EDITORS

RICHARD ARENS (Managing Editor)

University of California

Los Angeles, California 90024

C. W. Curtis

University of Oregon

Eugene, OR 97403

C. C. MOORE

J. DUGUNDJI

Department of Mathematics University of Southern Californa Los Angeles, California 90007

R. Finn AND J. Milgram Stanford University Stanford, California 94305

University of California

Berkeley, CA 94720

\section{ASSOCIATE EDITORS}

E. F. BeCKENBACH

B. H. NeUMANN

F. WOLF

K. YosHida

\section{SUPPORTING INSTITUTIONS}

UNIVERSITY OF BRITISH COLUMBIA UNIVERSITY OF SOUTHERN CALIFORNIA CALIFORNIA INSTITUTE OF TECHNOLOGY UNIVERSITY OF CALIFORNIA MONTANA STATE UNIVERSITY UNIVERSITY OF NEVADA, RENO STANFORD UNIVERSITY UNIVERSITY OF TOKYO UNIVERSITY OF UTAH NEW MEXICO STATE UNIVERSITY WASHINGTON STATE UNIVERSITY OREGON STATE UNIVERSITY UNIVERSITY OF WASHINGTON UNIVERSITY OF OREGON OSAKA UNIVERSITY 


\section{Pacific Journal of Mathematics \\ Vol. 73, No. 1 \\ March, 1977}

Thomas Robert Berger, Hall-Higman type theorems. $V \ldots \ldots \ldots \ldots \ldots \ldots \ldots$

Frank Peter Anthony Cass and Billy E. Rhoades, Mercerian theorems via

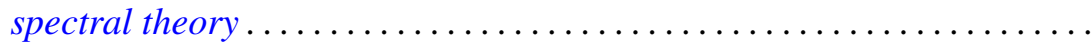

Morris Leroy Eaton and Michael David Perlman, Generating $\mathrm{O}(n)$ with

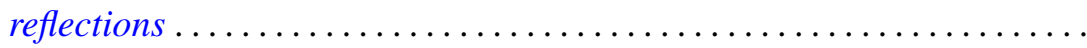

Frank John Forelli, Jr., A necessary condition on the extreme points of a

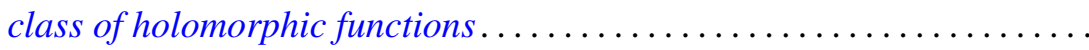

Melvin F. Janowitz, Complemented congruences on complemented

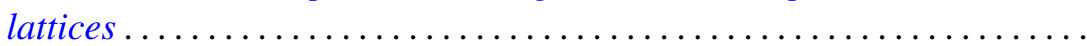

Maria M. Klawe, Semidirect product of semigroups in relation to amenability, cancellation properties, and strong $F \phi$ lner conditions....

Theodore Willis Laetsch, Normal cones, barrier cones, and the "spherical image" of convex surfaces in locally convex spaces ................

Chao-Chu Liang, Involutions fixing codimension two knots.............

Joyce Longman, On generalizations of alternative algebras .............

Giancarlo Mauceri, Square integrable representations and the Fourier

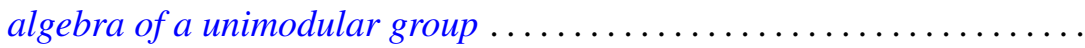

J. Marshall Osborn, Lie algebras with descending chain condition...

John Robert Quine, Jr., Tangent winding numbers and branched

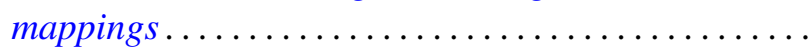

Louis Jackson Ratliff, Jr. and David Eugene Rush, Notes on ideal covers and associated primes .

H. B. Reiter and N. Stavrakas, On the compactness of the hyperspace of faces.

Walter Roth, A general Rudin-Carlson theorem in Banach-spaces ..

Mark Andrew Smith, Products of Banach spaces that are uniformly rotund in every direction.

Roger R. Smith, The R-Borel structure on a Choquet simplex ...

Gerald Stoller, The convergence-preserving rearrangements of real infinite series. ...

Graham H. Toomer, Generalized homotopy excision theorems modulo a Serre class of nilpotent groups...

Norris Freeman Weaver, Dehn's construction and the Poincaré conjecture....

Steven Howard Weintraub, Topological realization of equivariant intersection forms... 